\section{L'enfant, l'urbain et la société}

\section{Bassand}

L'enfant est un acteur, c'est dire qu'en fonction de sa position sociale et en relation avec d'autres (parents, camarades), il se construit une identité et des projets. La réalité urbaine (logements, voisinages, quartiers) constitue une dimension fondamentale de ce processus de formation de l'acteur-enfant.

\section{Problématique}

Le triangle de la figure 1 synthétise la problématique que nous allons préciser dans le texte qui suit.

\section{Figure 1}

L'enfant, un acteur social.

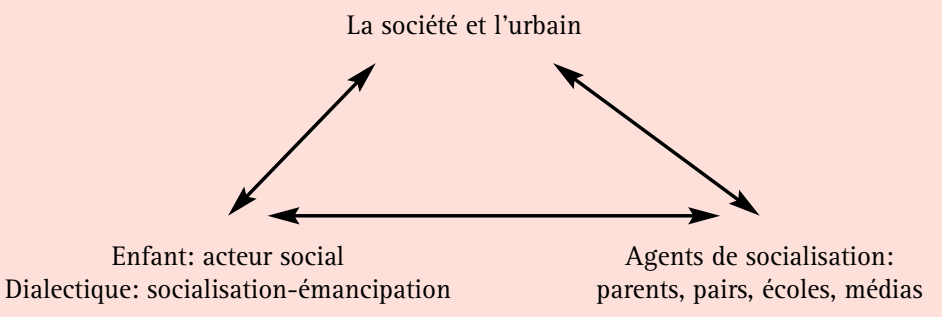

D'emblée, explicitons ce triangle:

- Le premier pôle, c'est l'enfant acteur social, qui dispose d'une autonomie, certes très variable. D'abord, elle lui est octroyée, puis, au fur et à mesure de sa croissance, il la conquiert pour arriver jusqu'à l'âge adulte. Cette conquête varie selon ses qualités psychophysiologiques, soit son âge et son sexe, mais aussi selon la position sociale que lui donnent ses parents: leur catégorie socioprofessionnelle (impliquant revenu et niveau d'instruction), leur culture (langue, religion, nationalité, etc.). Selon la combinaison de ces ingrédients, l'enfant s'émancipera plus ou moins rapidement

Correspondance:

Prof. Michel Bassand

École polytechnique fédérale de Lausanne (EPFL)

Institut de recherche sur l'environnement construit (IREC)

Avenue Eglise-Anglaise 14

Case postale 555

$\mathrm{CH}-1001$ Lausanne et habilement pour se façonner une position sociale propre et autonome. Elle sera entière à l'âge adulte. Ce dernier est défini différemment selon les milieux socioculturels: 18 ans? Service militaire? Fin de la scolarité? Première activité professionnelle? Bref, l'enfant, acteur social, peut être défini par une tension entre socialisation et émancipation. D'aucuns définissent l'enfance par la socialisation, c'est incontestable; cependant, dès le plus jeune âge, l'enfant se manifeste par une volonté d'émancipation qui s'exprime selon des modalités très différentes. Socialisation et émancipation sont indissociables, mais conditionnées socialement.

- Le deuxième pôle du triangle découle de ce que nous venons d'exposer: la socialisation de l'enfant est menée par tout un réseau d'agents. D'abord la mère et le père, auxquels sont associés d'autres parents (frères et sœurs, grands-parents, oncles et tantes), des amis et des voisins, etc. De nombreux professionnels interviennent également de manière décisive: pédiatre, prêtre, assistant social. L'enfant en bas âge est introduit dans sa société, c'est-à-dire socialisé, par des faits et gestes et des actions essentielles: communiquer, se mouvoir, être propre, etc. Au fur et à mesure des premiers mois, les agents de socialisation prendront des décisions, mais l'enfant commencera aussi à manifester les premiers mouvements d'émancipation, qui seront salués ou bien avec joie par les agents de socialisation, ou bien au contraire réprimés plus ou moins sévèrement. Ce chassé-croisé socialisation-émancipation se développera pendant toute l'enfance et l'adolescence, sous le contrôle et avec l'apparition de nouveaux agents: pairs, prêtres, éducateurs, enseignants, média, qui prendront le relais des premiers, surtout du père et de la mère. Ceux-ci se sentiront dépossédés de leur fonction de socialisation, parfois avec amertume.

- Venons-en au troisième pôle: la société et l'urbain. Ce n'est que tardivement que l'enfant découvrira que le réseau des agents de socialisation fait corps avec un immense et puissant système: d'abord l'urbain, puis la société tout entière, c'est-à-dire l'Etat (police, instituteur), mais aussi le marché, la culture, la religion, divers groupes et organisations (mouvements de jeunes, partis politiques, syndicats). Tous ont des rapports entre eux, mais aussi des conflits; enfin, la division du travail les met en correspondance. Progressivement et inégalement, selon le statut des parents, l'enfant sera initié à cette complexité et découvrira la puissance de la société, mais aussi peut-être apprendra-t-il qu'il est citoyen.

Ces trois pôles ne sont dissociables qu'à des fins analytiques, en fait ils forment un tout.

La suite de mon exposé consistera à préciser quelques-uns de ces aspects. Il faudrait plusieurs heures pour être complet. 


\section{La société du 21 e siècle?}

En fait, elle est déjà là, depuis dix à vingt ans! Depuis plusieurs années, nous agissons dans la société du 21e siècle.

Nous avons quasiment tous été socialisés dans une autre société, que les sociologues nomment société industrielle. En Suisse, elle a pris corps au début $\mathrm{du}$ 19e siècle avec l'industrialisation, la formation d'un Etat fédéral avec ses institutions politiques, scolaires, militaires. La société industrielle se manifeste encore par les processus de sécularisation et d'urbanisation. Ce n'est qu'en 1950-60, avec les indicateurs importants de la tertiairisation, qu'est posée la question d'un changement de société: on parle alors de société postindustrielle. Dès 1990, il n'est plus possible d'utiliser ce terme, car l'informatisation rapide et systématique s'empare de toutes les dimensions de la société. Nous sommes dans un système sociétal nouveau: la société informationnelle, etj'ajoute, programmée. Cette société informationnelle et programmée (SIP) n'a plus rien à voir avec la société industrielle [1], le changement est total. C'est d'ailleurs la raison pour laquelle d'aucuns parlent de chaos. Certes, des traces de l'ordre ancien subsistent encore ici et là. Explicitons quelques dimensions de cette SIP.

- D'abord, la technoscience. C'est la matrice culturelle des SIP, c'est elle qui donne du sens. Elle domine la religion, la langue et d'autres traits culturels tout en les intégrant. La science et la technique sont fort anciennes, en revanche, ce qui est nouveau, c'est leur couplage; elles sont devenues inséparables. L'exemple de technoscience le plus superbe est celui de la médecine, qui combine la technique et la science médicale, la pharmacie, l'hygiène et bien d'autres domaines. Un autre exemple concerne les sciences de l'information, l'informatique, la robotique, etc. La SIP est fondée sur cette technoscience qu'est l'informatisation.

- Une autre dimension essentielle de la SIP, c'est la mondialisation-globalisation. Elle change l'horizon de l'Homme. Il était principalement national, dans SIP il devient mondial. En même temps, l'économie de marché devient hégémonique: privatisations et dérégulations font que l'État est redimensionné, bien que toujours important. Mais ces changements entraînent de nouvelles inégalités sociales et exclusions, tant à l'échelle nationale que mondiale. Ajoutons enfin que la mondialisation suscite des réactions défensives de la part des collectivités locales et régionales. La mondialisation ne provoque pas l'uniformisation tant redoutée, à juste titre, par certains.

- Enfin, la SIP renforce une tendance déjà manifeste dans les sociétés industrielles: l'individualisation. Le marché, la consommation et la culture de masse, la démocratie et d'autres facteurs encore contribuent à promouvoir l'individu roi. Les groupes et autres collectivités tels que la famille, l'entreprise, la paroisse, le quartier, sont remis en cause. Il s'agit dès lors d'inventer de nouvelles structures collectives dont on se rend bien compte qu'elles sont indispensables. Le concept de réseau devient central. Il implique la mise en place de structures collectives le moins contraignantes possibles pour l'individu. La famille-réseau, l'entreprise-réseau, l'urbain en réseau sont devenus des stratégies fondamentales. Je ne vais pas allonger, mais, face à cette individualisation, la question se pose: comment allons-nous vivre ensemble au niveau local, national, mondial? Et, deuxième question: comment l'enfant va-t-il être socialisé, et comment va-t-il affirmer son indispensable émancipation dans un tel contexte?

\section{Mort de la ville et règne de l'urbain}

J'emprunte ce titre à la grande dame de l'urbanisme qu'est Françoise Choay [2] (1994). L'étalement urbain en termes de suburbanisation, de périurbanisation, de rurbanisation, fait éclater la ville, elle est remplacée par des agglomérations urbaines. F. Choay les baptise du néologisme l'urbain. Elles sont très diverses, mais elles sont dominées par les métropoles, c'est-à-dire des agglomérations urbaines de très grande taille (environ un million d'habitants et plus), ayant une centralité mondiale. En effet, le monde contemporain est dominé par un réseau mondial de métropoles [3]. Soyons précis: ce réseau mondial compte 400 métropoles, c'est-à-dire 400 agglomérations urbaines d'environ un million d'habitants. Ce réseau de 400 métropoles regroupe le $40 \%$ de la population urbaine du monde; il domine et dirige la mondialisation dont je vous ai dit précédemment l'importance. Ces agglomérations-métropoles organisent également la Suisse. Permettez-moi quelques chiffres fournis par le recensement fédéral de 1990. La Suisse contemporaine compte 48 agglomérations qui regroupent $70 \%$ de la population sur $17 \%$ du territoire. C'en est fini de la Suisse du peuple de bergers! Les Suisses sont d'abord des citadins. Ces 48 agglomérations urbaines sont dominées par les métropoles zurichoise, rhénane, lémanique et insubrica, ainsi que par la région urbaine du Plateau central. La Suisse est dès lors d'abord faite de ces cinq pôles métropolitains.

Faisons un pas de plus. L'urbain, ou l'agglomération-métropole, a une organisation ou une échelle spatiale bien précise, qui va de niveaux micro-urbains à des niveaux macro-urbains. Ainsi peuvent être distingués le logement, le voisinage, le quartier et la zone, la commune, l'agglomération urbaine, la métropole. Chacun de ces niveaux ou échelons a une organisation propre et, partant, une identité et un vécu, c'est-à-dire qu'ils influencent la vie quotidienne des citadins.

Ils sont agencés en fonction de deux principes: la spécialisation fonctionnelle $d u$ sol et la ségrégation sociale.

- La première consiste à regrouper les activités et équipements en zones homogènes: l'habitation en voisinages et quartiers, et les diverses activités en zones spécifiques: zones industrielles, administratives, gares, hôpitaux, universités, prisons, aéroports, parcs, etc. 
- Quant à la ségrégation, elle consiste à regrouper les diverses catégories de population (en termes de catégories socioprofessionnelles, de religions, langues, ethnies) dans des quartiers et voisinages spécifiques, si bien qu'elles vivent séparées les unes des autres. Les diverses banlieues françaises, par exemple, sont le résultat de la ségrégation, comme les quartiers noirs aux USA, les bidonvilles et quartiers à habitat précaire dans les métropoles du Sud. La ségrégation est une des causes de la violence urbaine. La ségrégation résulte des lois du marché du logement, mais aussi des affinités ou répulsions socioculturelles des divers habitants d'une société, qui entraînent la fragmentation de l'urbain.

Cette vaste mosaïque urbaine ne peut fonctionner que grâce à un système sophistiqué de réseaux d'eaux, d'énergie, de voies, de télécommunications, de transports. Ou encore, l'urbain ainsi divisé ne peut former un tout que si la population est mobile. Il s'agit-là d'un trait fondamental: un citadin non mobile est un citadin marginalisé, exclu.

Si j’ai précisé ces quelques caractéristiques de l'urbain, c'est qu'elles sont très importantes pour la socialisation et l'émancipation de l'enfant. Je l'expliciterai dans le paragraphe qui suit.

\section{L'enfant, un acteur social}

D’emblée, précisons ce terme d'acteur. Un acteur social peut être un individu ou un groupe. Restons-en au premier. L'individu est acteur lorsqu'il conquiert et dispose d'une autonomie, certes jamais totale, mais suffisante pour lui permettre d'agir sur son existence et sur la société. Cette autonomie est construite à partir de sa position sociale, qui implique l'âge, le genre, la catégorie socioprofessionnelle, les différentes facettes de la culture (religion, langue, nationalité, ethnie). Cette position sociale procure des ressources, façonne un intérêt, des valeurs qui permettent à l'individu de tisser un réseau de relations sociales qui peuvent être à leur tour des ressources ou des contraintes.

Une deuxième dimension de l'acteur, c'est l'identité qu'il se construit, mais toujours en relation avec autrui. L'identité, c'est-à-dire l'image de soi, est toujours construite dans les relations interpersonnelles: cette image peut être emblématique ou stigmate. L'identité donne sens à l'existence; selon qu'elle est positive ou négative, elle conditionne les pratiques de l'individu.

Enfin, l'acteur se définit par ses projets, qui eux aussi dépendent des rapports à autrui. Nous distinguons les projets de vie quotidienne des projets de société. Les deuxièmes sont toujours construits en commun.

La rationalité de l'acteur, c'est la modalité selon laquelle il aménage une cohérence entre, d'une part, les ressources, intérêts, valeurs qui dépendent de sa position sociale, d'autre part, son identité, et enfin ses projets. Il n'y a pas qu'une rationalité, mais chaque type d'acteur développe une rationalité spécifique. Il en va ainsi de l'enfant aux différentes phases de son développement et en fonction des différents milieux socioculturels dans lesquels il est plongé.

A partir de cette conception de l'enfant en tant qu'acteur social, reprenons quelques-unes des dimensions que j'ai précédemment mises en relief.

Par exemple l'individualisation. Elle donne naissance à un autre type familial: la famille monoparentale. Dans cette dernière, la socialisation et l'émancipation de l'enfant font intervenir une configuration d'agents de socialisation très différents de ceux de la famille nucléaire. L'émancipation est plus aisée, la socialisation moins rigoureuse.

Néanmoins, avec ses conséquences sur la famille, l'individualisation a pour corollaire un phénomène inquiétant pour les sociétés occidentales: la chute de la natalité. Nos sociétés ne réussissent plus à se reproduire par elles-mêmes, tant la natalité est faible. Pourquoi? Parce que le désir d'enfant s'est transformé radicalement. Dans les sociétés agraires préindustrielles, le nombre d'enfants servait des causes et fonctions multiples:

1. une main d'œuvre bon marché;

2. à cause de la mortalité infantile élevée, et afin d'être sûr d'avoir quelques enfants jusqu'en fin de vie, il fallait en concevoir beaucoup;

3. il était impérieux que quelques enfants subsistent, car c'étaient des "bâtons de vieillesse» qui pouvaient adoucir des fins de vie très dures;

4. enfin, l'enfant était une bénédiction divine, donc un signe du salut.

Depuis l'ère industrielle et la famille nucléaire, ces raisons d'avoir des enfants ont disparu: ce n'est plus de la main d'œuvre, la mortalité infantile a fortement diminué, la sécurité sociale - notamment l'assurance vieillesse - fonctionne plus ou moins bien, et l'on ne croit plus en Dieu. Par conséquent, il n'est plus utile d'avoir beaucoup d'enfants. Pour des raisons hédonistes, un ou deux suffisent, et surtout pas davantage, car non seulement ils ne servent plus, mais en revanche ils coûtent très cher! De mon point de vue, la fonction de l'enfant est l'explication de la chute de la natalité.

Pour ce qui est de l'urbain, considérons les niveaux de l'organisation de l'espace que sont le logement, le voisinage, le quartier, la commune, l'agglomération urbaine, la métropole [4]. Cette échelle, du moins les quatre premiers échelons, est très significative pour l'enfant, car ces quatre échelons marquent des phases précises de sa socialisation. D'abord, il est confiné à sa chambre et au logement, dont le style et l'organisation - partant les jouets - interfèrent grandement dans la socialisation et l'émancipation. Dès qu'il sait marcher, l'enfant va découvrir le voisinage, ses premiers copains, d'autres adultes que papa et maman. Puis il commencera à découvrir le quartier et ses équipements socioculturels variés: l'école, l'église, les magasins et des acteurs plus intransigeants: la police, les enseignants, les travail- 
leurs sociaux de différents types. Un peu plus âgé, accompagné, il découvre la commune, l'agglomération urbaine. Ce n'est que lorsqu'il maîtrisera la mobilité (vélo, transports en commun) qu'il explorera l'urbain dans son entier. C'est à ce stade qu'il s'émancipera de manière significative, grâce notamment à ces espaces où il échappe au contrôle des parents.

Par ces quelques touches, on se rend compte que l'environnement construit et l'urbain sont des instances importantes de socialisation, mais aussi d'émancipation.

Finalement, mentionnons un autre impact des transformations urbaines. Nous avons vu que la spécialisation fonctionnelle du sol contribue à créer des espaces différenciés. Notamment, elle distingue et sépare profondément les espaces d'habitation des espaces de travail. Pour aller de l'un à l'autre, l'individu y consacre un temps variable. Pour certains, ce facteur devient prohibitif, puisqu'ils ne peuvent plus rentrer à midi à la maison pour déjeuner. Pour ceux qui ont des enfants, cela signifie qu'ils ne les voient au mieux que le matin, à l'occasion d'un départ précipité, et le soir, quand tout le monde est fatigué et n'est plus bon qu'à regarder la TV. Dans ces conditions, comment le père peut-il assumer ses fonctions de socialisation? Le temps du week-end et des vacances est-il suffisant? Bien évidemment que non ...

\section{Conclusion}

Tout indique que la société informationnelle et programmée ainsi que l'urbain correspondent et contribueront à façonner l'enfant acteur social. Est-ce un bien ou un mal? Que pouvons-nous faire? Un travail immense nous attend, car la transformation de la société et de l'urbain nécessitent des moyens très coûteux et lents, qui passent par l'intermédiaire de processus politiques dont on connaît les aléas. Impossible de faire autrement. D'ailleurs, peut-on souhaiter une autre politique que celle que nous connaissons en Suisse? Immense question ...

\section{Références}

1 Bassand M. Métropolisation et inégalités sociales. Lausanne: PPUR; 1997.

2 Choay F. Le règne de l'urbain et la mort de la ville. Dans: La ville: art et architecture en Europe 1870-1933. Paris: Centre Pompidou; 1994. P. 26-35.

3 Ascher F. Métapolis. Paris: 0. Jacob; 1995.

4 Grafmeyer Y. Trajectoires familiales et espaces de vie en milieu urbain. Lyon: Presses universitaires de Lyon; 1998. 